TRANSACTIONS OF THE

AMERICAN MATHEMATICAL SOCIETY

Volume 191, 1974

\title{
ANALYTIC CENTERS AND ANALYTIC DIAMETERS OF PLANAR CONTINUA(1)
}

BY

\section{STEVEN MINSKER}

ABSTRACT. This paper contains some basic results about analytic centers and analytic diameters, concepts which arise in Vitushkin's work on rational approximation. We use Carathéodory's theorem to calculate $\beta(K, z)$ in the case in which $K$ is a continuum in the complex plane. This leads to the result that, if $g: \Omega(K) \rightarrow \Delta(0 ; 1)$ is the normalized Riemann map, then $\beta(g, 0) / \gamma(K)$ is the unique analytic center of $K$ and $\beta(K)=\gamma(K)$. We also give two proofs of the fact that $\beta(g, 0) / \gamma(K) \epsilon \operatorname{co}(K)$. We use Bieberbach's and Pick's theorems to obtain more information about the geometric location of the analytic center. Finally, we obtain inequalities for $\beta(E, z)$ for arbitrary bounded planar sets $E$.

I. Introduction. The concepts of analytic center and analytic diameter arose in the work of A. G. Vitushkin ([10], [11]), who found necessary and sufficient conditions, in terms of continuous analytic capacity, for certain planar function algebras to be coincident. Following Gamelin [3], we shall modify Vitushkin's original idea by defining analytic centers and analytic diameters in terms of analytic capacity rather than continuous analytic capacity. It is our purpose in this paper to completely discuss the case in which the planar set in question is compact and connected, and to shed some light on more general cases. As will become evident, many of the classical results from the theory of one complex variable come into play.

II. Preliminaries. Let $K$ be a compact subset of the complex plane. Let $\Omega(K)$ denote the open connected subset of the Riemann sphere $S^{2}$ obtained by taking the unbounded connected component of $K^{\sim}$ and adding the point at infinity. We say that a function $f: \Omega(K) \rightarrow C$ is admissible for $K$ if $f$ is analytic on $\Omega(K)$, $f(\infty)=0$, and $\sup _{z \in \Omega(K)}|f(z)| \leq 1$. Let $E$ be any subset of the complex plane. $A$ function is admissible for $E$ if it is admissible for some compact subset of $E$. We denote the set of admissible functions for $E$ by $\mathscr{Q}(E)$.

Received by the editors March 22, 1973 and, in revised form, July 2, 1973. AMS (MOS) subject classifications (1970). Primary 30 A34, 30A44.

(1) This paper is based on the author's Ph. D. thesis at the Massachusetts Institute of Technology, June 1972. 
Fix $z_{0} \in \mathrm{C}$. Any function $f$ which is analytic in a neighborhood of infinity has a series representation near infinity of the form

$$
f(z)=a_{0}+\frac{a_{1}}{z-z_{0}}+\frac{a_{2}}{\left(z-z_{0}\right)^{2}}+\frac{a_{3}}{\left(z-z_{0}\right)^{3}}+\cdots
$$

It is clear that $a_{0}=f(\infty)$. Also, $a_{1}$ is independent of the point $z_{0}$, since

$$
a_{1}=\lim _{z \rightarrow \infty} z(f(z)-f(\infty)) \text {. }
$$

Hence, we adopt the notation $a_{1}=f^{\prime}(\infty)$. The coefficient $a_{2}$ does depend on $z_{0}$, and so we write $a_{2}=\beta\left(f, z_{0}\right)$. For $R$ large,

$$
f^{\prime}(\infty)=\frac{1}{2 \pi i} \int_{|z|=R} f(z) d z \text { and } \beta\left(f, z_{0}\right)=\frac{1}{2 \pi i} \int_{|z|=R} f(z)\left(z-z_{0}\right) d z \text {. }
$$

Hence, for any two points $z_{1}, z_{2}$, we get

$$
\beta\left(f, z_{1}\right)=\beta\left(f, z_{2}\right)+\left(z_{2}-z_{1}\right) f^{\prime}(\infty) .
$$

Now if $E$ is as above, we define $\gamma(E)$, the analytic capacity of $E$, as $\gamma(E)=$ $\sup _{f \in \mathbb{R}(E)}\left|f^{\prime}(\infty)\right|$. We shall assume that the reader is familiar with the most elementary facts concerning analytic capacity; they may be found in [3, Chapter VIII] and [12, Chapter 3].

Theorem 1. Let $E$ be contained in the disc $\bar{\Delta}\left(z_{0} ; r\right)$ and let $f \in \mathbb{Q}(E)$. Then $\left|\beta\left(f, z_{0}\right)\right| \leq r \gamma(E)$.

Proof. If $f \in \mathbb{Q}(E)$, then $f \in \mathbb{Q}(K)$ for some compact $K \subset E$. Define $\phi(z)=$ $\left(z-z_{0}\right) f(z) / r$. Then $\phi$ is analytic on $\Omega(K)$, and $|\phi| \leq 1$ on $\Omega(K)$ by the maximum principle. If $\left|\phi\left(z^{\prime}\right)\right|=1$ for some $z^{\prime} \in \Omega(K)$, we would have $\phi \equiv \lambda$ for some complex constant $\lambda$ of modulus 1 ; but then $f(z)=\lambda_{r} /\left(z-z_{0}\right)$, and $\beta\left(f, z_{0}\right)=0$. So we can assume $|\phi|<1$ on $\Omega(K)$. Define

$$
b(z)=\frac{\phi(z)-\phi(\infty)}{1-\overline{\phi(\infty)} \phi(z)} .
$$

It is then immediate that $b \in \mathbb{Q}(K)$, so $\left|b^{\prime}(\infty)\right| \leq \gamma(E)$. But $b^{\prime}(\infty)=\phi^{\prime}(\infty) /\left(1-|\phi(\infty)|^{2}\right)$ $=\beta\left(f, z_{0}\right) / r\left(1-|\phi(\infty)|^{2}\right)$. Since $|\phi(\infty)|<1$, we get $\left|\beta\left(f, z_{0}\right)\right| \leq r \gamma(E)$, completing the proof.

From now on, $E$ will denote a bounded subset of the complex plane with $\gamma(E)>0$.

Definition. For each $z \in \mathrm{C}$, let $\beta(E, z)=\sup _{f \in \mathbb{Q}(E)}|\beta(f, z)| / \gamma(E)$ and $M(E, z)=\sup _{x \in E}|x-z|$.

With this notation, our previous theorem may be stated as $\beta(E, z) \leq M(E, z)$ for all $z$. In particular, we may note that $\beta(E, z) \leq \operatorname{diam} E$ for $z \in E$.

Our equation $(*)$ easily yields the following three basic facts, the proofs of which are omitted. 
Theorem 2. For arbitrary $z_{1}, z_{2} \in \mathrm{C}$ :

(A) $\left|\beta\left(E, z_{1}\right)-\beta\left(E, z_{2}\right)\right| \leq\left|z_{1}-z_{2}\right|$. So $\beta(E, z)$ is a continuous function of $z$.

(B) $\beta\left(E, z_{1}\right)+\beta\left(E, z_{2}\right) \geq\left|z_{1}-z_{2}\right|$. So $\beta(E, z) \rightarrow \infty$ as $z \rightarrow \infty$.

(C) If $f \in \mathbb{Q}(E)$, and $0 \leq \lambda \leq 1$, then $\beta\left(f, \lambda z_{1}+(1-\lambda) z_{2}\right)=\lambda \beta\left(f, z_{1}\right)+$ $(1-\lambda) \beta\left(f, z_{2}\right)$. So $\beta(E, z)$ is a convex function of $z$.

Definition. The analytic diameter $\beta(E)$ of the set $E$ is defined as $\beta(E)=$ $\inf _{z \in C} \beta(E, z)$. Let $E^{\prime}=\{\omega \in \mathrm{C}: \beta(E, \omega)=\beta(E)\}$. By Theorem 2, it follows that $E^{\prime}$ is a nonempty compact convex set. Any point $\omega \in E^{\prime}$ is called an analytic center of $E$.

Theorem 3. $\gamma(E) \leq \beta(E) \leq \operatorname{diam} E$.

Proof. If $z_{0} \in E$, then $\beta(E) \leq \beta\left(E, z_{0}\right) \leq \operatorname{diam} E$. For the first assertion, suppose we are given $\gamma(E)>\epsilon>0$. Take $f \in \mathbb{Q}(E)$ with $f^{\prime}(\infty)>\gamma(E)-\epsilon$. Then $f^{2} \in \mathbb{Q}(E)$ and

$$
\beta(E, z) \geq \frac{\left|\beta\left(f^{2}, z\right)\right|}{\gamma(E)}=\frac{\left(f^{\prime}(\infty)\right)^{2}}{\gamma(E)}>\frac{(\gamma(E)-\epsilon)^{2}}{\gamma(E)}
$$

for all $z$. Letting $\epsilon \rightarrow 0$, we get $\beta(E, z) \geq \gamma(E)$. Hence $\beta(E) \geq \gamma(E)$.

The following facts are all easy and immediate consequences of our first two theorems. Again we omit proofs.

Fact 1. For all $z \in \mathbf{C}$ and $\omega \in E^{\prime}$

$$
\max \{\beta(E),|z-\omega|-\beta(E)\} \leq \beta(E, z) \leq|z-\omega|+\beta(E) .
$$

Fact 2. For all $z, M\left(E^{\prime}, z\right) \leq 2 M(E, z)$. In particular,

$$
\sup \left\{|z-\omega|: z \in E, \omega \in E^{\prime}\right\} \leq 2 \operatorname{diam} E,
$$

so every analytic center must be "close" to every point of $E$.

Fact 3. If $E \subset \bar{\Delta}(a ; r)$, then $E^{\prime} \subset \bar{\Delta}(a ; 2 r)$ and $\beta(E) \leq r$.

We conclude this section with some transparent results on translation, magnification, rotation, and symmetry.

Fact 4. For any $c, z_{0}, z \in \mathbf{C}$ with $c \neq 0$, we have

(1) $\beta(E, z)=\beta\left(E+z_{0}, z+z_{0}\right)$.

(2) $\beta(c E, c z)=|c| \beta(E, z)$.

Fact 5 . For any $c, z_{0}, z \in \mathbf{C}$ with $c \neq 0$, we have

(1) $\beta(E)=\beta\left(E+z_{0}\right)$ and $\left(E+z_{0}\right)^{\prime}=E^{\prime}+z_{0}$.

(2) $\beta(c E)=|c| \beta(E)$ and $(c E)^{\prime}=c \cdot E^{\prime}$.

Fact 6. If $E$ is symmetric with respect to a line, then so are $\beta(E, z)$ and $E^{\prime}$. In particular, since $E^{\prime}$ is convex, $E$ has an analytic center lying on the line.

Fact 7. If $E$ is symmetric with respect to two distinct lines, their intersection is an analytic center of $E$. (So there is at least some geometric justification for the term "center" being used.) 
III. The case for continua. Throughout this section, $K$ will be a compact connected subset of the complex plane (to avoid trivialities, we assume that $K$ is not a one-point set), and $g: \Omega(K) \rightarrow \Delta(0 ; 1)$ will be the normalized Riemann map (by "normalized", we mean $\left.g^{(\infty)}=0, g^{\prime(\infty)}>0\right)$. It is well known that $g^{\prime}(\infty)=\gamma(K)$.

We shall be needing a specific case of the following classical result. For an elegant proof using abstract Hardy space theory, as well as a discussion of the classical sources, see [8].

Theorem (Carathéodory). Given $n+1$ complex numbers $c_{0}, c_{1}, \cdots, c_{n}$, there exists an analytic function $\phi: \Delta(0 ; 1) \rightarrow \bar{\Delta}(0 ; 1)$ whose power series begins with the terms $c_{0}+c_{1} z+\cdots+c_{n} z^{n}$ if and only if the matrix

$$
\left[\begin{array}{lllll}
c_{0} & 0 & 0 & \cdots & 0 \\
c_{1} & c_{0} & 0 & \cdots & 0 \\
c_{2} & c_{1} & c_{0} & \cdots & 0 \\
\cdot & \cdot & \cdot & & \\
\cdot & \cdot & \cdot & & \\
\cdot & \cdot & \cdot & & \\
c_{n} & c_{n-1} & c_{n-2} & \cdots & c_{0}
\end{array}\right]
$$

bas norm $\leq 1$ as an operator on the Hilbert space $\mathbf{C}^{n+1}$.

We shall now calculate $\beta(K, z)$ for all $z$.

Theorem 4 .

$$
\beta(K, z)=\left\{\begin{array}{l}
\gamma(K)+\frac{1}{4 \gamma(K)}\left|z-\frac{\beta(g, 0)}{\gamma(K)}\right|^{2} \text { for }\left|z-\frac{\beta(g, 0)}{\gamma(K)}\right| \leq 2 \gamma(K) \\
\left|z-\frac{\beta(g, 0)}{\gamma(K)}\right| \text { for }\left|z-\frac{\beta(g, 0)}{\gamma(K)}\right|>2 \gamma(K)
\end{array}\right\} \text {. }
$$

Proof. Let $K^{*}=K-\beta(g, 0) / \gamma(K)$. If $b(z)=g(z+\beta(g, 0) / \gamma(K))$, then $b: \Omega\left(K^{*}\right) \rightarrow \Delta(0 ; 1)$ is the normalized Riemann map, and so $\gamma(K)=g^{\prime}(\infty)=b^{\prime}(\infty)=$ $\gamma\left(K^{*}\right)$. Using equation $(*)$ preceding Theorem 1 , we get

$$
\beta(h, 0)=\beta\left(g, \frac{\beta(g, 0)}{\gamma(K)}\right)=\beta(g, 0)-\frac{\beta(g, 0)}{\gamma(K)} g^{\prime}(\infty)=0 .
$$

Let $c_{1}, c_{2} \in \mathrm{C}$. Now there exists $f \in \mathbb{Q}\left(K^{*}\right)$ with $f^{\prime}(\infty)=c_{1}$ and $\beta(f, 0)=c_{2}$ if and only if there exists an analytic function $\phi: \Delta(0 ; 1) \rightarrow \Delta(0 ; 1)$ whose power series begins with the terms $c_{1} z / \gamma\left(K^{*}\right)+c_{2} z^{2} /\left(\gamma\left(K^{*}\right)\right)^{2}$. (The correspondence is evidently $\phi=f \circ b^{-1}$.) By Carathéodory's theorem, this last condition is equivalent to the matrix 


$$
M=\left[\begin{array}{ccc}
0 & 0 & 0 \\
\frac{c_{1}}{\gamma\left(K^{*}\right)} & 0 & 0 \\
\frac{c_{2}}{\left(\gamma\left(K^{*}\right)\right)^{2}} & \frac{c_{1}}{\gamma\left(K^{*}\right)} & 0
\end{array}\right]
$$

having norm $\leq 1$ as an operator on $C^{3}$. Since $\|M\|^{2}$ equals the maximum eigenvalue of the operator $M^{*} M$ (where $M^{*}$ is the conjugate transpose of $M$ ), it is easy to see that

$$
\|M\|=\left(|A|^{2}+1 / 2|B|^{2}+1 / 2|B| \sqrt{4|A|^{2}+|B|^{2}}\right)^{1 / 2},
$$

where $A=c_{1} / \gamma\left(K^{*}\right)$ and $B=c_{2} /\left(\gamma\left(K^{*}\right)\right)^{2}$. The condition $\|M\| \leq 1$ is thus seen to be equivalent to the condition $|A|^{2}+|B| \leq 1$, that is, $\left|c_{1}\right|^{2}+\left|c_{2}\right| \leq\left(\gamma\left(K^{*}\right)\right)^{2}$. So, using equation $(*)$ again, we get

$$
\begin{aligned}
\beta\left(K^{*}, z\right) & =\sup _{f \in Q\left(K^{*}\right)} \frac{|\beta(f, z)|}{\gamma\left(K^{*}\right)}=\sup _{f \in Q\left(K^{*}\right)} \frac{\left|\beta(f, 0)-z f^{\prime}(\infty)\right|}{\gamma\left(K^{*}\right)} \\
& =\frac{1}{\gamma\left(K^{*}\right)} \sup \left\{\left|c_{2}-z c_{1}\right|:\left|c_{1}\right|^{2}+\left|c_{2}\right| \leq\left(\gamma\left(K^{*}\right)\right)^{2}\right\} \\
& =\frac{1}{\gamma\left(K^{*}\right)} \sup \left\{\left|c_{2}\right|+|z|\left|c_{1}\right|:\left|c_{1}\right|^{2}+\left|c_{2}\right| \leq\left(\gamma\left(K^{*}\right)\right)^{2}\right\} \\
& =\frac{1}{\gamma\left(K^{*}\right)} \max \left\{\left(\gamma\left(K^{*}\right)\right)^{2}-x^{2}+|z| x: 0 \leq x \leq \gamma\left(K^{*}\right)\right\} \\
& =\left\{\begin{array}{l}
\gamma\left(K^{*}\right)+\frac{1}{4 \gamma\left(K^{*}\right)}|z|^{2} \text { for }|z| \leq 2 \gamma\left(K^{*}\right) \\
|z| \text { for }|z|>2 \gamma\left(K^{*}\right)
\end{array}\right\} .
\end{aligned}
$$

By Fact 4 , however, $\beta(K, z)=\beta\left(K^{*}, z-\beta(g, 0) / \gamma(K)\right)$, and we already know $\gamma\left(K^{*}\right)$ $=\gamma(K)$. Our result then follows.

Corollary 5. $\beta(K)=\gamma(K)$, and $\beta(g, 0) / \gamma(K)$ is the unique analytic center of $K$. Corollary 6.

$$
\beta(\Delta(a ; r), z)=\left\{\begin{array}{l}
r+\frac{1}{4 r}|z-a|^{2} \text { for }|z-a| \leq 2 r \\
|z-a| \text { for }|z-a|>2 r
\end{array}\right\} .
$$

Hence $\beta(\bar{\Delta}(a ; r))=r$ and $a$ is the unique analytic center of $\bar{\Delta}(a ; r)$.

Proof. If $K=\bar{\Delta}(a ; r)$, then $g(z)=r /(z-a)$. For $|z|>|a|, g(z)=r / z+a r / z^{2}+$ $a^{2} r / z^{3}+\cdots$, and so $g^{\prime}(\infty)=\gamma(K)=r$ and $\beta(g, 0)=a r$. The result follows. 
More generally, if $K$ is doubly symmetric (in the sense of Fact 7), Corollary 5 now tells us that the geometric center of $K$ is the unique analytic center.

Remark. If $K_{1}, K_{2}$ are as in the beginning of this section, with $g_{1}, g_{2}$ their associated Riemann maps, and if $K_{1} \subset K_{2}$ and $\beta\left(g_{1}, 0\right) / \gamma\left(K_{1}\right)=\beta\left(g_{2}, 0\right) / \gamma\left(K_{2}\right)$, then Theorem 4 implies $\beta\left(K_{1}, z\right) \leq \beta\left(K_{2}, z\right)$ for all $z$. The assumption that the analytic centers coincide is necessary. For instance, if we take $K_{1}=\bar{\Delta}(0 ; 1)$ and $K_{2}=\bar{\Delta}(1 ; 3)$, then Corollary 6 implies $\beta\left(K_{1}, 8\right)=8>7=\beta\left(K_{2}, 8\right)$. In particular, this shows that a statement made in [3, Chapter VIII, $\$ 6]$ is false.

Remark. Since it is well known that the transfinite diameter, logarithmic capacity, and analytic capacity of a continuum are all equal, we have shown the equivalence of the analytic diameter and transfinite diameter for such sets. Whether or not they are equivalent in general is, to the best of my knowledge, an open question.

For the rest of this section, let $\omega=\beta(g, 0) / \gamma(K)$.

Theorem 7. $\omega \in \operatorname{co}(K)$.

First proof. Let $\bar{\Delta}(z ; r)$ be any closed disc containing $K$. Then Theorem 4 and Theorem 1 imply $|z-\omega| \leq \beta(K, z) \leq M(K, z) \leq r$, and so $\bar{\Delta}(z ; r)$ contains $\omega$. Since $\bar{\Delta}(z ; r)$ was arbitrary and $c o(K)$ is compact, the result follows.

Second proof. Let $\hat{K}$ denote the union of $K$ and the bounded components of $K^{\sim}$, and let $\partial(\hat{K})$ be its boundary. We shall first prove the theorem under the assumption that $\partial(\hat{K})$ is a Jordan curve. The map $g$ then extends to a homeomorphism $\tilde{g}: \overline{\Omega(K)} \rightarrow \bar{\Delta}(0 ; 1)$. We claim that

$$
\omega=\frac{1}{2 \pi} \int_{0}^{2 \pi}(\widetilde{g})^{-1}\left(e^{i \theta}\right) d \theta
$$

Since $\lim _{z \rightarrow 0} z g^{-1}(z)=\gamma(K)$ and $g^{-1}$ is analytic in $0<|z|<1$, it follows that $g^{-1}$ has Laurent expansion around zero of the form $g^{-1}(z)=\gamma(K) / z+\alpha_{0}+\alpha_{1} z+$ $a_{2} z^{2}+\cdots$, valid for all $0<|z|<1$ and converging uniformly on compact subsets. If $0<r<1$, term-by-term integration implies

$$
a_{0}=\frac{1}{2 \pi i} \int_{|z|=r} \frac{g^{-1}(z)}{z} d z
$$

Letting $r \rightarrow 1$, we get

$$
a_{0}=\frac{1}{2 \pi i} \int_{|z|=1} \frac{(\tilde{g})^{-1}(z)}{z} d z=\frac{1}{2 \pi} \int_{0}^{2 \pi}(\tilde{g})^{-1}\left(e^{i \theta}\right) d \theta .
$$

But

$$
\begin{aligned}
\beta(g, 0) & =\lim _{\zeta \rightarrow \infty} \zeta^{2} g(\zeta)-\zeta \gamma(K)=\lim _{z \rightarrow 0}\left(g^{-1}(z)\right)^{2} z-\gamma(K) g^{-1}(z) \\
& =\lim _{z \rightarrow 0} z g^{-1}(z)\left[g^{-1}(z)-\frac{\gamma(K)}{z}\right]=\gamma(K) a_{0}
\end{aligned}
$$


so $a_{0}=\omega$. This establishes our claim $(* *)$. Hence, $\omega$ is a limit of expressions of the form

$$
\sum_{j=1}^{n}(\tilde{g})^{-1}\left(e^{\left.i \theta_{j}^{*}\right)} \frac{\left(\theta_{j}-\theta_{j-1}\right)}{2 \pi}, \quad \theta_{j-1} \leq \theta_{j}^{*} \leq \theta_{j}, \theta_{0}=0, \theta_{n}=2 \pi,\right.
$$

each of which is a convex linear combination of points in $K$ (in fact, in $\partial(\hat{K})$ ). So $\omega \in \overline{\mathrm{co}}(K)=\operatorname{co}(K)$.

Now, for arbitrary $K$, there exists a sequence of compact, connected, simply connected sets $K_{n}$ such that $K_{n} \supset K_{n+1}, \bigcap K_{n}=\hat{K}$, and, for each $n, \partial K_{n}$ is a Jordan curve. Let $g_{n}$ and $\omega_{n}$ be the normalized Riemann map and unique analytic center associated with $K_{n}$. By the first part of this proof, $\omega_{n} \in \operatorname{co}\left(K_{n}\right)$. It is well known that $g_{n} \rightarrow g$ uniformly on compact subsets of $\Omega(K)$. Using the integral representations for $g_{n}^{\prime}(\infty)$ and $\beta\left(g_{n}, 0\right)$ established earlier, it follows that $\gamma\left(K_{n}\right)$ $\rightarrow \gamma(K)$ and $\beta\left(g_{n}, 0\right) \rightarrow \beta(g, 0)$. So $\omega_{n} \rightarrow \omega$. Since co $\left(K_{n}\right)$ is compact and $\operatorname{co}\left(K_{n}\right) \supset \operatorname{co}\left(K_{n+1}\right)$, we have $\omega \in \operatorname{co}\left(K_{n}\right)$ for all $n$. Then $\omega \in \bigcap \operatorname{co}\left(K_{n}\right)=\operatorname{co}(\hat{K})=$ co $(K)$. This completes the second proof.

We can get some further information about the geometric location of $\omega$ by using two classical results concerning univalent functions. Let $\mathcal{F}$ denote the collection of all univalent analytic functions $f: \Delta(0 ; 1) \rightarrow C$ with $f(0)=0$ and $f^{\prime}(0)=1$. Such functions have power series expansions of the form

$$
f(z)=z+a_{2} z^{2}+a_{3} z^{3}+\cdots \cdot
$$

Bieberbach's theorem states that $\left|a_{2}\right| \leq 2$ whenever $f \in \mathcal{F}$. Pick's theorem states that $\left|a_{2}\right| \leq 2(1-1 / M)$ whenever $f \in \mathcal{F}$ and $|f| \leq M$.

Theorem 8. $\sup \{|z-\omega|: z \in K\} \leq 2 \gamma(K)$.

Proof. Fix $z_{0} \in K$, and let $f(z)=\gamma(K) /\left(g^{-1}(z)-z_{0}\right)$. Then $f \in \mathcal{F}$, so $\left|a_{2}\right| \leq 2$. But

$$
\begin{aligned}
a_{2} & =\lim _{z \rightarrow 0} \frac{f(z)-z}{z^{2}}=\lim _{\zeta \rightarrow \infty} \frac{f(g(\zeta))-g(\zeta)}{(g(\zeta))^{2}}=\lim _{\zeta \rightarrow \infty} \frac{z_{0} \zeta g(\zeta)-\left(\zeta^{2} g(\zeta)-\zeta \gamma(K)\right)}{\left(\zeta^{2}-\zeta z_{0}\right)\left(g(\zeta)^{2}\right.} \\
& =\frac{z_{0} \gamma(K)-\beta(g, 0)}{(\gamma(K))^{2}}=\frac{1}{\gamma(K)}\left(z_{0}-\omega\right) .
\end{aligned}
$$

Thus $\left|z_{0}-\omega\right| \leq 2 \gamma(K)$, establishing the result.

Corollary 9. If $z \in K$, then

$$
\beta(K, z)=\gamma(K)+\frac{1}{4 \gamma(K)}|z-\omega|^{2} .
$$

Hence $\beta(K, z) \leq 2 \gamma(K)$ for all $z \in K$. 
Proof. Both statements follow immediately from Theorem 4 and Theorem 8.

Example. A compact, connected, simply connected set need not contain its analytic center. Pick $\epsilon>0$ but small and let

$$
K=\left\{r e^{i \theta}: 0 \leq r \leq 1, \theta= \pm \arcsin \epsilon\right\} .
$$

We shall be very lazy in that we shall not try to exhibit the map g. By Fact $6, \omega$ is real. For any $\delta>0$, there is a curve $\Gamma$ winding around $K$ such that length $(\Gamma)<2+2 \epsilon+\delta$, so $\gamma(K) \leq(2+2 \epsilon) / 2 \pi=(1+\epsilon) / \pi$. Theorem 8 implies that $\omega$ must be within $2 \gamma(K)$ of every point of $K$. Since $0 \epsilon K$ and $\sqrt{1-\epsilon^{2}}+i \epsilon \epsilon K$, we respectively obtain $\omega \leq 2(1+\epsilon) / \pi$ and $\omega \geq \sqrt{1-\epsilon^{2}}-\sqrt{4(1+\epsilon)^{2} / \pi^{2}-\epsilon^{2}}$. So $1-2 / \pi-$ $\nu(\epsilon)<\omega<2 / \pi+\nu(\epsilon)$, where $\nu(\epsilon) \rightarrow 0$ as $\epsilon \rightarrow 0$. In particular, $\omega \notin K$ for all small $\epsilon$.

Theorem 10. Let $K_{i}, g_{i}, \omega_{i}$ be as usual for $i=1$, 2. If $K_{1} \subset K_{2}$, then $\left|\omega_{2}-\omega_{1}\right| \leq 2\left(\gamma\left(K_{2}\right)-\gamma\left(K_{1}\right)\right)$.

Proof. Since $\Omega\left(K_{2}\right) \subset \Omega\left(K_{1}\right)$, we can define $\phi: \Delta(0 ; 1) \rightarrow \Delta(0 ; 1)$ by $\phi=g_{1} \circ$ $g_{2}^{-1}$. Then $\phi$ is a univalent analytic function with $\phi(0)=0$. If $\phi(z)=a_{1} z+a_{2} z^{2}$ $+\cdots$, an application of Pick's theorem to $\phi / a_{1}$ yields $\left|a_{2} / a_{1}\right| \leq 2\left(1-\left|a_{1}\right|\right)$. But

$$
a_{1}=\lim _{z \rightarrow 0} \frac{\phi(z)}{z}=\lim _{\zeta \rightarrow \infty} \frac{g_{1}(\zeta)}{g_{2}(\zeta)}=\lim _{\zeta \rightarrow \infty} \frac{\zeta g_{1}(\zeta)}{\zeta g_{2}(\zeta)}=\frac{\gamma\left(K_{1}\right)}{\gamma\left(K_{2}\right)},
$$

and

The result then follows.

$$
\begin{aligned}
a_{2} & =\lim _{z \rightarrow 0} \frac{\phi(z)-a_{1} z}{z^{2}}=\lim _{\zeta \rightarrow \infty} \frac{g_{1}(\zeta)-a_{1} g_{2}(\zeta)}{\left(g_{2}(\zeta)\right)^{2}} \\
& =\lim _{\zeta \rightarrow \infty} \frac{\left(\zeta^{2} g_{1}(\zeta)-\zeta \gamma\left(K_{1}\right)\right)+a_{1}\left(\zeta \gamma\left(K_{2}\right)-\zeta^{2} g_{2}(\zeta)\right)}{\left(\zeta g_{2}(\zeta)\right)^{2}} \\
& =\frac{\beta\left(g_{1}, 0\right)-a_{1} \beta\left(g_{2}, 0\right)}{\left(\gamma\left(K_{2}\right)\right)^{2}}=\frac{\gamma\left(K_{1}\right)}{\left(\gamma\left(K_{2}\right)\right)^{2}}\left(\omega_{1}-\omega_{2}\right) .
\end{aligned}
$$

Corollary 11. If $K \subset \bar{\Delta}(a ; r)$, then $|a-\omega| \leq 2(r-\gamma(K))$.

Proof. Take $K_{1}=K$ and $K_{2}=\bar{\Delta}(a ; r)$ in the theorem, and use Corollary 6 .

IV. Remarks about the general case. Needless to say, once we abandon the assumption that we are working with compact connected sets, problems about analytic centers and analytic diameters become extremely difficult, since we no longer have available Riemann maps to convert these problems into questions about the open unit disc. We shall therefore content ourselves with a result about "nice" open sets, and three inequalities that follow from Carathéodory's theorem. 
We say a compact set $K$ in the complex plane is starlike with respect to $a \in$ int $K$ if $(a, x) \subset$ int $K$ whenever $x \in K$. Note that such sets are connected and simply connected.

Theorem 12. If $K$ is starlike (with respect to some $a \in$ int $K$ ), then $\beta$ (int $K, z)=\beta(K, z)$ for all $z$.

Proof. By Fact 4, we can assume $a=0$. If $0<r<1$, then $r K \subset$ int $K$, so

$$
\gamma(K) \geq \gamma(\text { int } K) \geq \sup _{0<r<1} \gamma(r K)=\sup _{0<r<1} r \gamma(K)=\gamma(K) \text {. }
$$

Thus $\gamma(K)=\gamma($ int $K)$. Since $\mathfrak{Q}($ int $K) \subset \mathbb{Q}(K)$, we have $\beta($ int $K, z) \leq \beta(K, z)$ for all $z$. For the reverse inequality, fix $z \in \mathbf{C}$ and $0<r<1$. Note that $f(\zeta) \in \mathbb{Q}(K)$ if and only if $b(\zeta) \equiv f(\zeta / r) \in \mathbb{Q}(r K)$, and $r^{2} \beta(f, z)=\beta(b, r z)$. Since $\mathbb{Q}(r K) \subset$ $\mathbb{Q}($ int $K$ ), we have

$$
\beta(K, z)=\sup _{f \in \mathbb{Q}(K)} \frac{|\beta(f, z)|}{\gamma(K)} \leq \sup _{b \in \mathbb{Q}(\text { int } K)} \frac{|\beta(b, r z)| / r^{2}}{\gamma(\text { int } K)}=\frac{1}{r^{2}} \beta(\text { int } K, r z) .
$$

By Theorem $2 \mathrm{~A}, \beta($ int $K, z)$ is a continuous function of $z$. So $\beta(K, z) \leq$ $\lim _{r \rightarrow 1} \beta($ int $K, r z) / r^{2}=\beta$ (int $\left.K, z\right)$, completing the proof.

Remark. In view of this result, and the fact that $\gamma($ int $K)=\gamma(K)$, all of the results of the previous section carry over in obvious fashion to the interiors of starlike sets (with the analogue of Theorem 7 being that $\omega \epsilon \overline{\text { co }}$ (int $K$ )).

An analysis of the proof of Theorem 4 yields the following version of Carathéodory's theorem, which is really all we need in dealing with analytic centers and analytic diameters:

Theorem. Let $b_{1}, b_{2} \in \mathbf{C}$. Then there exists an analytic function $\phi: \Delta(0 ; 1)$ $\rightarrow \Delta(0 ; 1)$ whose power series begins with the terms $b_{1} z+b_{2} z^{2}$ if and only if $\left|b_{1}\right|^{2}+\left|b_{2}\right| \leq 1$.

Theorem 13. Let $Q$ be a compact subset of the complex plane with $\gamma(Q)>0$. Let $b$ be the Ablfors function for $Q$, and let $\omega=\beta(b, 0) / \gamma(Q)$. Then

$$
\beta(Q, z) \geq\left\{\begin{array}{l}
\gamma(Q)+\frac{1}{4 \gamma(Q)}|z-\omega|^{2} \quad \text { for }|z-\omega| \leq 2 \gamma(Q) \\
|z-\omega| \text { for }|z-\omega|>2 \gamma(Q)
\end{array}\right\} \text {. }
$$

Proof. As in Theorem $4, \beta(b, \omega)=0$. If $|z-\omega| \leq 2 \gamma(Q)$, let

$$
b_{1}=-\frac{\bar{z}-\bar{\omega}}{2 \gamma(Q)}, \quad b_{2}=\frac{4(\gamma(Q))^{2}-|z-\omega|^{2}}{4(\gamma(Q))^{2}} .
$$

Then $\left|b_{1}\right|^{2}+\left|b_{2}\right|=1$. Let $\phi$ be as in our special version of Carathéodory's theorem, and let $f=\phi \circ b$. Then $f \in \mathbb{Q}(Q)$ with 


$$
\begin{aligned}
\beta(f, z) & =b_{1} \beta(b, z)+b_{2} \beta\left(b^{2}, z\right)=b_{1}(\omega-z) \gamma(Q)+b_{2}(\gamma(Q))^{2} \\
& =(\gamma(Q))^{2}+1 / 4|z-\omega|^{2} .
\end{aligned}
$$

So $\beta(Q, z) \geq|\beta(f, z)| / \gamma(Q)=\gamma(Q)+|z-\omega|^{2} / 4 \gamma(Q)$. If $|z-\omega|>2 \gamma(Q)$, let $f=$ $-(\bar{z}-\bar{\omega}) b /|z-\omega|$. Then $f \in \mathbb{Q}(Q)$, and $\beta(f, z)=-(\bar{z}-\bar{\omega}) \beta(b, z) /|z-\omega|=|z-\omega| \gamma(Q)$. So $\beta(Q, z) \geq|\beta(f, z)| / \mathcal{\gamma}(Q)=|z-\omega|$, establishing the theorem.

Remark. Our choice of $b_{1}$ and $b_{2}$ above was obviously motivated by an analysis of the maximizing function in Theorem 4.

Corollary 14. $\omega \in \operatorname{co}(Q)$.

Proof. Completely mimic the first proof of Theorem 7.

Remark. In view of Theorem 13 and Corollary 14, it would seem very likely that $\omega$ is an analytic center of $Q$, and probable that $\omega$ is the unique analytic center. Further evidence comes from the fact that $\omega$ must lie on any axis of symmetry of $Q$ (this follows from the uniqueness of the Ahlfors function), so, if $Q$ is doubly symmetric (in the sense of Fact 7), $\omega$ is indeed an analytic center of $Q$. But these two conjectures are still unresolved.

For the remainder of this paper, $E$ will be (as before) a bounded set with $\gamma(E)>0$.

Theorem 15. If $E \subset \bar{\Delta}(a ; r)$, then

$$
\beta(E, z) \leq\left\{\begin{array}{ll}
\frac{1}{\gamma(E)}\left(r^{2}+1 / 4|z-a|^{2}\right) & \text { for }|z-a| \leq 2 r \\
\frac{r}{\gamma(E)}|z-a| & \text { for }|z-a|>2 r
\end{array}\right\} \text {. }
$$

Proof. By Fact 4, we can assume that $a=0, r=1$. If $f \in \mathbb{Q}(E)$ with $f^{\prime}(\infty)=c_{1}$ and $\beta(f, 0)=c_{2}$, then there exists an analytic function $\phi: \Delta(0 ; 1) \rightarrow \Delta(0 ; 1)$ such that $\phi(z)=c_{1} z+c_{2} z^{2}+\cdots$ (Obviously, $\phi(z) \equiv f(1 / z)$.) By Carathéodory's theorem, we must have $\left|c_{1}\right|^{2}+\left|c_{2}\right| \leq 1$. But then

$$
\begin{aligned}
\beta(E, z) & =\sup _{f \in Q(E)} \frac{|\beta(f, z)|}{\gamma(E)}=\sup _{f \in \mathbb{Q}(E)} \frac{\left|\beta(f, 0)-z f^{\prime}(\infty)\right|}{\gamma(E)} \\
& \leq \frac{1}{\gamma(E)} \sup \left\{\left|c_{2}-z c_{1}\right|:\left|c_{1}\right|^{2}+\left|c_{2}\right| \leq 1\right\} \\
& =\left\{\begin{array}{ll}
\frac{1}{\gamma(E)}\left(1+1 / 4|z|^{2}\right) & \text { for }|z| \leq 2 \\
\frac{1}{\gamma(E)}|z| & \text { for }|z|>2
\end{array}\right\}
\end{aligned}
$$


Theorem 16. If $E \supset \bar{\Delta}(a ; r)$, then the reverse of the inequality in Theorem 15 bolds.

Proof. Completely analogous to that of Theorem 15.

Unfortunately, these last two estimates are far too weak to be of much value. In particular, they yield no new information about the location of analytic centers.

In closing, I shall list what I believe are the most important open questions in the field. They are as follows:

(1) Does $\beta(E)=\gamma(E)$ for all $E$ ?

(2) Does $E_{1} \subset E_{2} \Rightarrow \beta\left(E_{1}\right) \leq \beta\left(E_{2}\right)$ ?

(3) Does every set $E$ have a unique analytic center?

(4) Is it true that $E^{\prime} \subset \overline{c o}(E)$ ?

\section{BIBLIOGRAPHY}

1. C. Carathéodory, Über den Variabilitatsbereich der Koeffizienten von Potenzreihen, die gegebene Werte nicht annehmen, Math. Ann. 64 (1907), 95-115.

2. W. Fenchel, Convex cones, sets, and functions, Princeton Univ. Dept. of Math. Logistics Research Project, 1953.

3. T. W. Gamelin, Uniform algebras, Prentice-Hall, Englewood Cliffs, N. J., 1969.

4. E. Hille, Analytic function theory. Vol. II, Introduction to Higher Math., Ginn, Boston, Mass., 1962. MR 34 \#1490.

5. Z. Nehari, Conformal mapping, McGraw-Hill, New York, 1952. MR 13, 640.

6. G. Pick, Über die konforme Abbildung eines Kreises auf ein schlichtes und zugleich beschränktes Gebiet, S.-B. Kai serl. Akad. Wiss. Wien Math.-Natur. Kl. Abt. IIa 126 (1917), 247-263.

7. W. Rudin, Real and complex analysis, McGraw-Hill, New York, 1966. MR 35 \#1420.

8. D. Sarason, Generalized interpolation in $H^{\infty}$, Trans. Amer. Math. Soc. 127 (1967), 179-203. MR 34 \#8193.

9. L. Sario and K. Oikawa, Capacity functions, Die Grundlehren der math. Wissenschaften, Band 149, Springer-Verlag, New York, 1969. MR 40 \#741.

10. A. G. Vituškin, Necessary and sufficient conditions on a set in order that any continuous function analytic at the interior points of the set may admit of uniform approximation by rational functions, Dokl. Akad. Nauk SSSR 171 (1966), 1255-1258 = Soviet Math. Dokl. 7 (1966), 1622-1625. MR 35 \#391.

11. - Analytic capacity of sets and problems in approximation theory, Uspehi Mat. Nauk 22 (1967), no. 6 (138), 141-199 = Russian Math. Surveys 22 (1967), no. 6, 139200. MR 37 \#5404.

12. L, Zalcman, Analytic capacity and rational approximation, Lecture Notes in Math., no. 50, Springer-Verlag, Berlin and New York, 1968. MR 37 \#3018.

DEPARTMENT OF MATHEMATICS, UNIVERSITY OF PITTSBURGH, PITTSBURGH, PENNSYLVANIA 15213 